\title{
Effet D'un Extrait Aqueux De Pseudarthria Hookeri Wight \& Arn. (Fabaceae) Sur La Glycemie Et Sur La Liberation Et Le Stockage Du Glucose Hepatique De Rats Diabetiques
}

\author{
Gohi Parfait Kahou Bi \\ Kouakou Jean Claude Abo \\ Jean Severin Irie Bi
}

Laboratoire de Physiologie Animale Pharmacologie et Phytothérapie, UFR Biosciences, Université Félix Houphouët-Boigny, Abidjan, Côte d’Ivoire

\begin{abstract}
Pseudarthria hookeri (Fabaceae) is a plant used in traditional medicine in Ivory Coast to treat diabetes. This study aims to evaluate the antidiabetic effects of the aqueous extract of Pseudarthria hookeri (EAPh) on the release and storage of hepatic glucose in alloxan induced diabetic rats. The administration of EAPh, at a dose of $1200 \mathrm{mg} / \mathrm{kg}$ body weight for 28 days, leads to a significant decrease of glycaemia in diabetic rats. The oral administration of EAPh, at doses of $800 \mathrm{mg} / \mathrm{kg}, 1000 \mathrm{mg} / \mathrm{kg}$ and $1200 \mathrm{mg} / \mathrm{kg}$ body weight, reduced for $60 \mathrm{~min}$, in a dose-dependent manner, the release of hepatic glucose in normoglycemic rats. Moreover, for 90 days of treatment, EAPh significantly promotes the storage of hepatic glucose in diabetic rats. It appears that the aqueous extract of Pseudarthria hookeri has antidiabetic properties and acts by promoting the storage of hepatic glucose (glycogenesis), and by inhibiting the release of glucose from the liver (glycogenolysis). These results justify the use of Pseudarthria hookeri in traditional medicine to effectively treat diabetes.
\end{abstract}

Keywords: Diabetes mellitus, Pseudarthria hookeri, alloxan, glyceamia, glycogenesis, glycogenolysis

\section{Resume}

Pseudarthria hookeri (Fabaceae) est une plante utilisée en médecine traditionnelle en Côte d'Ivoire pour traiter le diabète. Cette étude vise à évaluer les effets antidiabétiques d'un extrait aqueux de Pseudarthria hookeri (EAPh) sur la glycémie et sur la libération et le stockage du glucose 
hépatique de rats rendus diabétiques par l'alloxane. L'administration de EAPh à la dose de 1200 mg/kg PC entraîne, après 28 jours de traitement, une baisse significative de la glycémie des rats rendus diabétiques. EAPh, aux doses de $800 \mathrm{mg} / \mathrm{kg}$ PC, $1000 \mathrm{mg} / \mathrm{kg}$ PC et $1200 \mathrm{mg} / \mathrm{kg}$ PC, réduit pendant 60 minutes, de façon dose-dépendante, la libération du glucose hépatique des rats normoglycémiques. Par ailleurs, après 90 jours de traitement, EAPh favorise de manière significative le stockage du glucose hépatique chez les rats rendus diabétiques. Il apparaît ainsi, que l'extrait aqueux de Pseudarthria hookeri a des propriétés antidiabétiques et agirait en favorisant le stockage du glucose hépatique (glycogénogenèse) et en inhibant la libération du glucose du foie (glycogénolyse). Ces résultats justifient l'utilisation en médecine traditionnelle de Pseudarthria hookeri pour traiter efficacement le diabète.

Mots-clés: Diabète, Pseudarthria hookeri, alloxane, glycémie, glycogénogenèse, glycogénolyse

\section{Introduction}

Le stockage des carbohydrates et des acides gras se fait sous forme de glycogène ou de triglycérides dans les tissus périphériques (Claude Bernard, 1853 ; Moussard, 2006). Ce mécanisme est sous le contrôle de l'insuline qui régule la glycémie. Cependant, un déséquilibre métabolique lié à un déficit ou une absence de sécrétion de l'insuline peut survenir et provoquer le diabète sucré. Dans le monde, on a dénombré 366 millions de personnes diabétiques avec 3,2 millions de décès par an (Whiting, 2011). En Côte d'Ivoire, suite à des enquêtes ethnobotaniques, Pseudarthria hookeri (Fabaceae) a été identifiée comme l'une des plantes utilisées pour traiter le diabète sucré (Ambe, 2000). Pseudarthria hookeri (Fabaceae) est une herbacée de 2 à $3 \mathrm{~m}$ de haut qui pousse dans les régions tropicales et subtropicales d'Afrique. Le but de cette étude est d'évaluer les effets d'un extrait aqueux de Pseudarthria hookeri (Fabaceae) sur la glycémie et sur la libération et le stockage du glucose hépatique chez les rats rendus diabétiques par l'administration d'alloxane.

\section{Materiel et methodes}

\section{Matériel végétal}

Il est constitué de feuilles fraîches de Pseudarthria hookeri, Wight \& Arn. (Fabaceae) récoltées dans la région de la Marahoué, au centre-ouest de la Côte d'Ivoire. Cette plante a été identifiée au Centre National de Floristique de l'Université Félix Houphouët-Boigny (Abidjan, Côte d'Ivoire). 


\section{Matériel animal}

Des rats blancs, Rattus norvegicus (Muridae), de souche Wistar, dont le poids varie entre 150 et 200 g, sont utilisés pour cette étude. Ils sont élevés dans l'animalerie de l'Unité de Formation et de Recherche (UFR) de Biosciences de l’Université Félix Houphouët-Boigny (Abidjan, Côte d’Ivoire). La salle reçoit un éclairage naturel avec une température moyenne de de $25^{\circ} \mathrm{C}$. Ces animaux sont nourris à volonté avec un aliment fabriqué par la société IVOGRAIN ${ }^{\circledR}$ d'Abidjan (Côte d'Ivoire), avec un accès libre à l'eau de robinet.

\section{Préparation de l'extrait aqueux de Pseudarthria hookeri (EAPh)}

Pour la préparation de l'extrait aqueux de Pseudarthria hookeri, 250 g de feuilles fraîches de cette plante, découpées en morceaux, sont portés à ébullition pendant 1 heure, dans 1,5 litre d'eau distillée. Le décocté obtenu est filtré deux fois sur du coton hydrophile et une fois sur du papier filtre Wattman $\mathrm{n}^{\circ} 1$. Le filtrat recueilli est séché à l'étuve à $50{ }^{\circ} \mathrm{C}$ pendant 72 heures. Après séchage, l'extrait aqueux total de Pseudarthria hookeri (EAPh) se présente sous forme de poudre. Cet extrait est quotidiennement administré, à différentes doses, par gavage aux rats en vue de déterminer ses effets pharmacologiques.

\section{Préparation de la solution de Mac Ewen}

Un litre de solution de Mac Ewen est composé de $130 \mathrm{mM}$ de $\mathrm{NaCl}$; 5,63mM de $\mathrm{KCl} ; 12,16 \mathrm{mM}$ de $\mathrm{CaCl}_{2} ; 0,91 \mathrm{mM}$ de $\mathrm{H}_{2} \mathrm{PO}_{4} \mathrm{Na} ; 11,90 \mathrm{mM}$ de $\mathrm{HCO}_{3} \mathrm{Na}$ et $0,25 \mathrm{mM}$ de $\mathrm{Mgcl}_{2} 2 \mathrm{~g}$ de glucose sont ajoutés à cette solution physiologique avant les expérimentations. Le Mac Ewen glucosé est utilisé pour l'étude de la libération du glucose du foie isolé de rat.

\section{Produits chimiques et pharmacologiques}

L’Alloxane (Alfa Aesar, Germany) est un diabétogène dont l'administration par voie intrapéritonéale détruit les cellules $\beta$ des ilots de Langerhans et induit le diabète expérimental. Le glibenclamide (Sanofi aventis, France) est une substance antidiabétique (sulfamide hypoglycémiant) qui stimule la sécrétion de l’insuline par le pancréas.

\section{Mesure du glucose libéré par le foie des rats normoglycémiques Principe}

En présence de glucose oxydase (GOD), le glucose est oxydé en acide gluconique. L’eau oxygénée libérée au cours de la réaction réagit sous l'action de la peroxydase (POD) avec le phénol et l'amino-4-phenazole pour former un complexe rose. L’intensité de la coloration est proportionnelle à la concentration de glucose de l'échantillon. 


\section{Protocole expérimental}

Cette étude est réalisée sur 25 rats Wistar normoglycémiques, de poids corporel (PC) compris entre 150 et 200 g, repartis en 5 lots. Les animaux du lot 1 (témoin) reçoivent par gavage de l'eau distillée durant 28 jours (durée de l'expérimentation). Ceux des lots 2, 3 et 4 sont traités avec l'extrait aqueux de Pseudarthria hookeri (EAPh), aux doses respectives de $800 \mathrm{mg} / \mathrm{kg}$ PC, $1000 \mathrm{mg} / \mathrm{kg}$ PC et $1200 \mathrm{mg} / \mathrm{kg}$ PC. Les rats du lot $5 \mathrm{sont}$ traités avec $10 \mathrm{mg} / \mathrm{kg}$ PC de glibenclamide. Après 28 jours de traitement, les animaux sont sacrifiés et un fragment de foie pesant $2 \mathrm{~g}$ est prélevé sur chacun des rats de chaque lot. Les fragments de foie recueillis dans les lots 1 , 2, 3, 4 et 5 sont plongés respectivement dans les solutions S1, S2, S3, S4, S5 contenant $4 \mathrm{ml}$ de Mac-Ewen glucosé, puis porté à incubation à $37 \mathrm{C}$ pendant 60 minutes. Le surnagent de chaque solution est prélevé pour doser, en présence du glucose GOD-POD (réactif), la quantité de glucose. Ce dosage est effectué à l'aide d'un spectrophotomètre (Biolabo, France), à 500 nm, aux temps 0 min (avant la mise en solution des organes), puis $10 \mathrm{~min}, 20$ min, 30 min, 40 min, 50 min et 60 min après l'immersion des organes dans la solution de Mac Ewen glucosé.

Etude des effets de l'extrait aqueux de Pseudarthria hookeri (EAPh) sur la glycémie de rats diabétiques

\section{Mesure de la glycémie}

La glycémie des rats rendus diabétiques est mesurée à l'aide d'un glucomètre de marque Accu-Chek Active et des bandelettes réactives (Roche diagnostic, Germany). Dans cette étude, les rats sont mis à jeun pendant 12 heures avant les expériences. Les substances à tester leur sont administrées par voie orale.

\section{Protocole expérimental}

Pour cette étude, 20 rats Wistar sont repartis en 4 lots. Leur poids varie entre 150 et 200 g. Le lot 1 est le témoin normoglycémique qui reçoit de l'eau distillée. Le lot 2 constitue le témoin diabétique recevant de l'eau distillée. Les lots 3 et 4 sont les lots de rats diabétiques traités respectivement avec 1200 mg/kg PC de EAPh et le glibenclamide à la dose de 10 mg/kg PC. L'expérimentation dure 28 jours et la glycémie est mesurée au temps $\mathrm{J}_{\mathrm{O}}$ (avant le début du gavage), puis 7, 14, 21 et 28 jours après le gavage.

\section{Mesure du glucose stocké par le foie des rats diabétiques Principe}

Le dosage du glucose stocké par le foie se fait en présence du réactif du glucose GOD-POD. 


\section{Protocole expérimental}

Cette étude est réalisée sur 20 rats Wistar repartis en 4 lots. Leur poids varie entre 150 et 200 g. Le lot 1 est le témoin normoglycémique qui reçoit de l'eau distillée. Le lot 2 constitue le témoin diabétique recevant de l'eau distillée. Les lots 3 et 4 sont les lots des rats diabétiques traités respectivement avec $1200 \mathrm{mg} / \mathrm{kg}$ PC de EAPh et le glibenclamide à la dose de $10 \mathrm{mg} / \mathrm{kg}$ PC. Après 90 jours de traitement, les animaux sont sacrifiés et un lobe pesant $5 \mathrm{~g}$ de foie est prélevé sur chacun des rats de chaque lot, découpé en petits morceaux, puis broyé dans $30 \mathrm{ml}$ d'acide trichloroacétique $4 \%$. Le broyat obtenu est mis dans un tube à essai et centrifugé à 4500 tour/min pendant $5 \mathrm{~min}$, puis le surnageant est récupéré. De l’éthanol $95 \%$ est ensuite ajouté au surnageant (éthanol/surnageant, 2v/v), le mélange est agité et chauffé dans un bain marie, lentement, jusqu’à ébullition. Le glycogène précipite et la suspension obtenue est refroidie et centrifugée à 4500 tour/min pendant $10 \mathrm{~min}$. Au culot (glycogène précipité) sont ajoutés 2 ml d'acide sulfurique $\left(\mathrm{H}_{2} \mathrm{SO}_{4}\right)$ à $2,5 \mathrm{~N}$ et le tube est chauffé pendant 30 minutes. Cette étape permet l'hydrolyse du glycogène en glucose. Après l'hydrolyse, le tube est refroidi et on y ajoute 1 goutte de dinitrophénolphtaléine, puis de la soude à 2,5 N jusqu'à l'obtention d'une coloration qui vire au rouge-rose. Cette étape permet de neutraliser l'acidité de l'hydrolysat. Pour chaque échantillon, le glucose ainsi formé est dosé, en présence du réactif GOD-POD (Trinder, 1969), par la méthode colorimétrique de Beer (1852). Le taux de glucose est déterminé à l'aide d'un spectrophotomètre (Biolabo, France), à 500 nm.

\section{Méthodes d'analyse statistique et traitements des résultats}

L’analyse des données est faite grâce au logiciel GraphPadInstat (San Diego CA, USA). Les résultats sont donnés sous forme de moyenne suivie de l'erreur standard sur la moyenne $(\mathrm{M} \pm \mathrm{ESM})$. La différence entre deux valeurs est déterminée par le test de Student-Newman-Keuls et est considérée comme significative pour $\mathrm{P}<0,05$. Le logiciel GraphPadPrism (San Diego CA, USA) est utilisé pour tracer les graphiques.

\section{Résultats}

\section{Effets de l'extrait aqueux de Pseudarthria hookeri (EAPh) sur le glucose hépatique libéré par le foie des rats normoglycémiques}

La concentration de glucose de chacune des solutions de Mac Ewen glucosé, avant l'expérience, est $2 \pm 0,01 \mathrm{~g} / \mathrm{L}$ (Figure 1). La concentration de glucose de la solution contrôle (S1) contenant le foie des rats témoins ayant reçu de l'eau distillée passe de $2 \pm 0,01 \mathrm{~g} / \mathrm{L}$, au début de l'expérience, à 2,40 $\pm 0,02 \mathrm{~g} / \mathrm{L}$, à la fin de l'expérience; soit $20 \%$ d'augmentation du taux de glucose. Les concentrations de glucose des solutions S2, S3 et S4, qui ont 
reçu le foie des rats traités avec EAPh aux doses respectives de $800 \mathrm{mg} / \mathrm{kg}$ PC, $1000 \mathrm{mg} / \mathrm{kg}$ PC et $1200 \mathrm{mg} / \mathrm{kg}$ PC, sont de 2,37 $\pm 0,02 \mathrm{~g} / \mathrm{L} ; 2,30 \pm 0,02$ $\mathrm{g} / \mathrm{L}$ et 2,23 \pm 0,03 g/L respectivement à la fin de l'expérimentation. Dans ces solutions, le taux de glucose augmente donc de 18,5 \% (S2), 15 \% (S3) et $11,5 \%$ (S4) par rapport à la concentration initiale de glucose. Dans la solution S5 qui contient le foie des rats traités avec le glibenclamide à la dose de $10 \mathrm{mg} / \mathrm{kg} \mathrm{PC}$, la concentration de glucose à la fin de l'expérimentation est 2,22 \pm 0,02 g/L ; soit $11 \%$ d’augmentation par rapport à la concentration initiale de glucose. L'évolution du taux de glucose hépatique dans la solution S2 est sensiblement égale à celle du taux de glucose dans la solution S1. Cependant, comparativement au taux de glucose de la solution contrôle S1, les concentrations en glucose des solutions S3, S4, S5 ont diminué significativement $(\mathrm{P}<0,05)$. En effet, à la fin de l'expérimentation, le taux de glucose est réduit de 4,17 \% (S3) ; 7,08 \% (S4) et 7,5 \% (S5), comparativement à la solution contrôle S1.

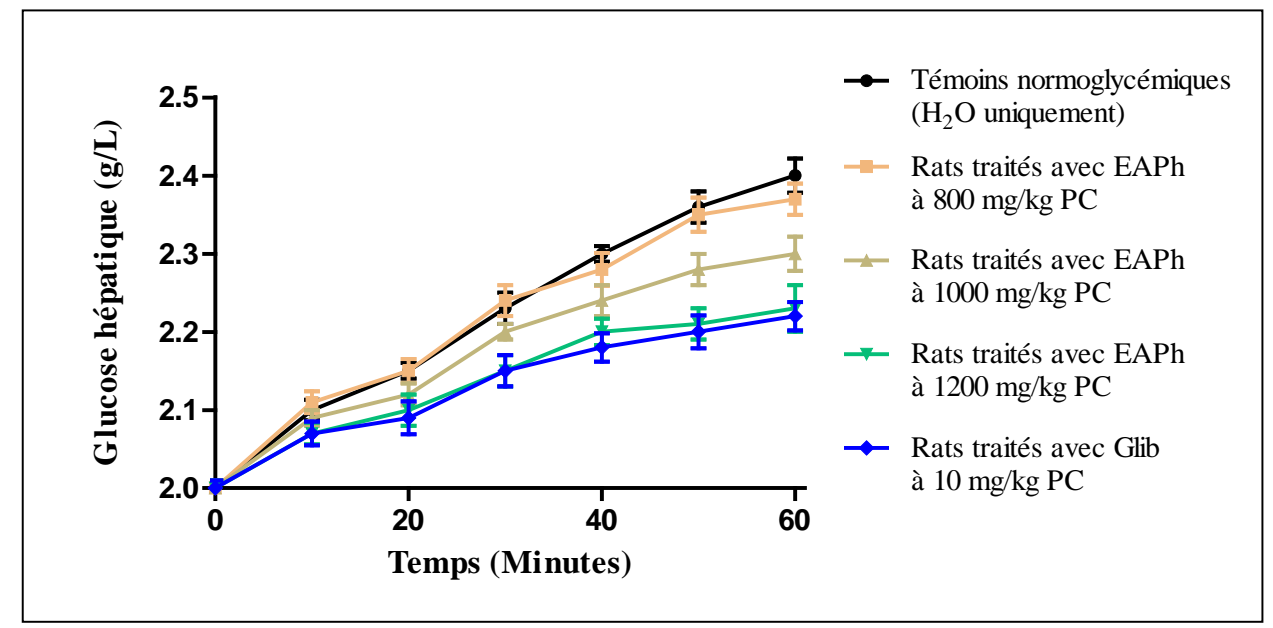

Figure 1 : Effets dose-réponse de l'extrait aqueux de Pseudarthria hookeri (EAPh) et du glibenclamide (Glib) sur la libération du glucose hépatique de rats normoglycémiques

\section{Effets de l'extrait aqueux de Pseudarthria hookeri (EAPh) sur la glycémie de rats diabétiques}

La glycémie ne présente aucune variation significative $(\mathrm{P}>0,05)$ chez les rats témoins normaux non traités (témoins normoglycémiques). Elle est de l'ordre de 1,02 $\pm 0,05 \mathrm{~g} / \mathrm{L}$ durant les 28 jours d'expérimentation (Figure 2). Après l'injection intrapéritonéale de l'alloxane monohydrate aux rats, leur glycémie passe de 1,02 $\pm 0,05 \mathrm{~g} / \mathrm{L}$ à 2,01 $\pm 0,06 \mathrm{~g} / \mathrm{L}$; soit une augmentation de glycémie de 0,99 \pm 0,07 g/L (97,06 \%) lorsque les rats sont rendus diabétiques. Chez les rats rendus diabétiques et qui ne sont pas traités (rats témoins diabétiques), la glycémie ne varie pas significativement ( $\mathrm{P}>$ 0,05 ) durant les 28 jours d'expérimentation. Elle est de l'ordre de 2,10 \pm 0,09 
g/L. Lorsque les rats rendus diabétiques sont traités avec EAPh à la dose de $1200 \mathrm{mg} / \mathrm{kg}$ PC, après 7 jours de traitement, leur glycémie n'est pas différente de celle des témoins diabétiques $(\mathrm{P}>0,05)$. Mais, à partir du $14^{\mathrm{ème}}$ jour, la glycémie baisse significativement $(\mathrm{P}<0,01)$ en fonction du temps. Ainsi, après le $28{ }^{\text {ème }}$ jour de traitement, la glycémie n'est plus que de $1,40 \pm$ $0,08 \mathrm{~g} / \mathrm{L}$ (37,25 \% d'augmentation de la glycémie, par rapport à celle des rats témoins normaux); soit une réduction de 59,81 \% de l'hyperglycémie induite par l'alloxane, lorsque les rats diabétiques sont traités avec EAPh. Après une semaine d'administration de glibenclamide à la dose de $10 \mathrm{mg} / \mathrm{kg} \mathrm{PC}$, la glycémie des rats diabétiques traités baisse significativement $(P<0,01)$. Cette réduction d'hyperglycémie augmente progressivement et, au $28^{\text {ème }}$ jour, la glycémie n'est plus que de 1,20 \pm 0,06 g/L (17,65 \% d'augmentation de la glycémie, par rapport à celle des rats témoins normaux); soit une réduction de 79,41 \% de l'hyperglycémie induite par l'alloxane, lorsque les rats diabétiques sont traités avec le glibenclamide.

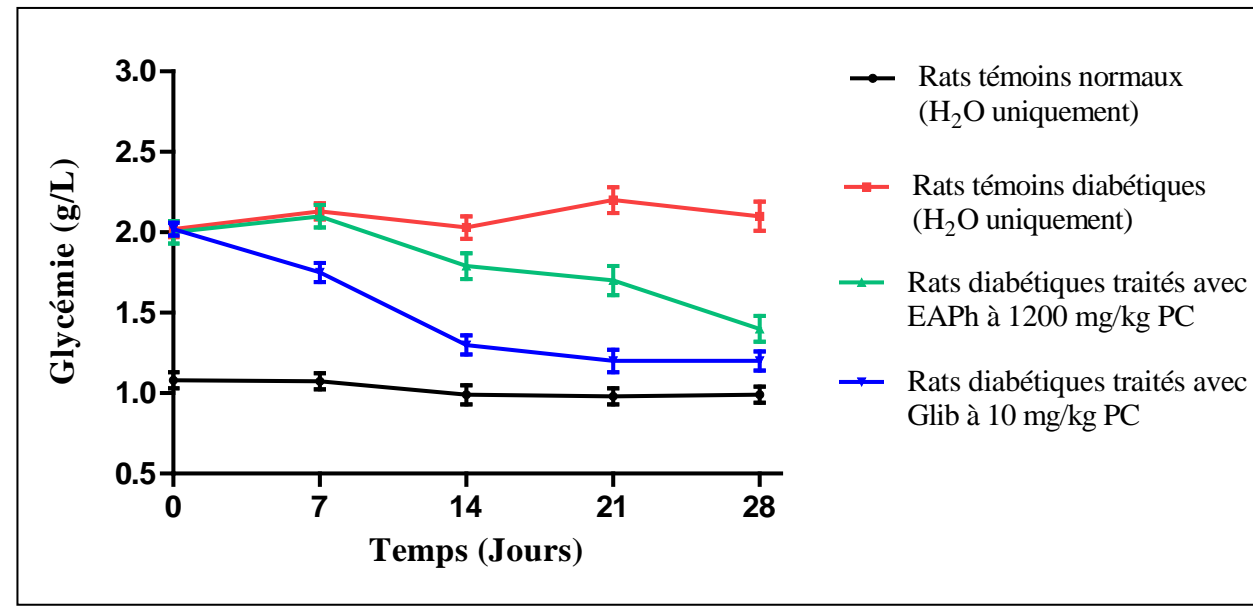

Figure 2 : Effets de l'extrait aqueux de Pseudarthria hookeri (EAPh) et du glibenclamide (Glib) sur la glycémie de rats diabétiques

\section{Effets de l'extrait aqueux de Pseudarthria hookeri (EAPh) sur le glucose hépatique stocké par le foie de rats diabétiques}

A la fin de l'expérience qui a duré 90 jours, chez les rats témoins normaux (rats témoins non traités), le taux de glucose hépatique stocké est $0,52 \pm 0,06$ g/L (Figure 3). Par contre, chez les rats témoins diabétiques, le taux de glucose hépatique stocké est $0,26 \pm 0,06 \mathrm{~g} / \mathrm{L}$; soit une diminution du taux de glucose hépatique stocké de $50 \%(\mathrm{P}<0,001)$ par rapport à celui des rats témoins normaux. Lorsque les animaux diabétiques sont traités avec EAPh à la dose de 1200 mg/kg PC ou le glibenclamide à la dose de $10 \mathrm{mg} / \mathrm{kg}$ PC, les taux de glucose hépatique stocké mesurés sont respectivement 0,47 \pm $0,05 \mathrm{~g} / \mathrm{L}$ et $0,49 \pm 0,04 \mathrm{~g} / \mathrm{L}$; soient, des augmentations de taux de glucose 
hépatique stocké respectivement de 80,77 \% et 88,46 \% (P < 0,001). Ainsi, comparativement aux rats témoins sains, lorsque les rats diabétiques sont traités avec EAPh ou le glibenclamide, les taux de glucose hépatique stocké mesurés sont sensiblement identiques $(\mathrm{P}>0,05)$.

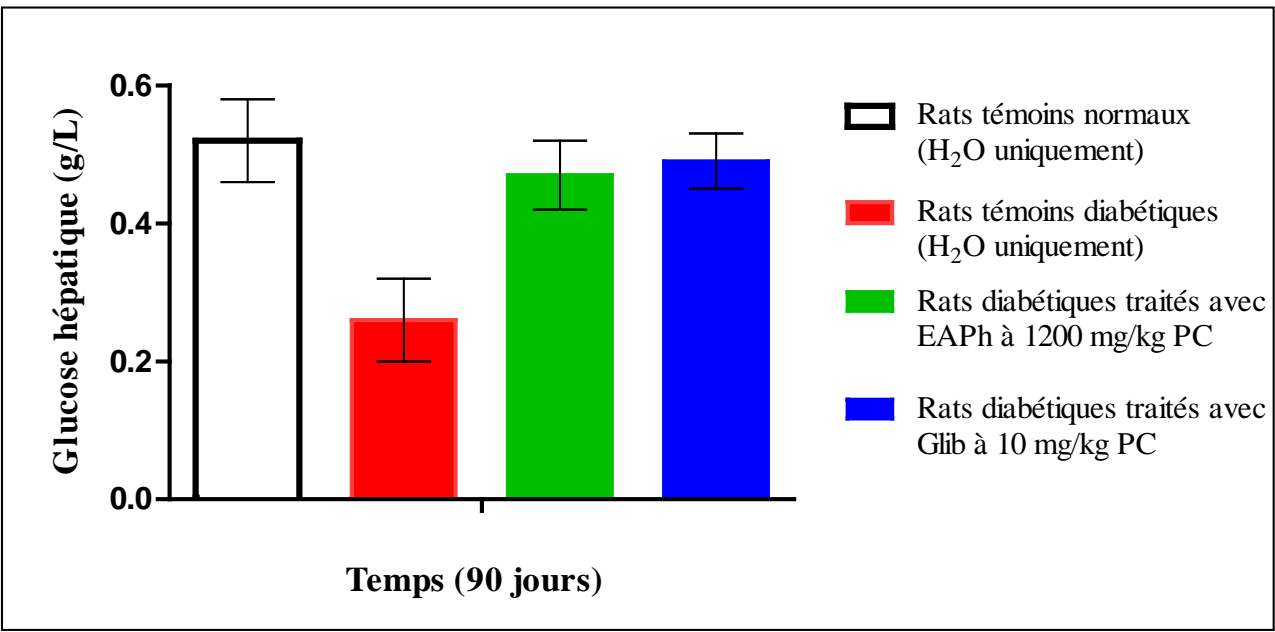

Figure 3 : Effets de l'extrait aqueux de Pseudarthria hookeri (EAPh) et du glibenclamide (Glib) sur le stockage du glucose hépatique de rats

\section{Discussion}

Le taux de glucose libéré par le foie de rats normoglycémiques témoins et des rats normoglycémiques traités avec EAPh ou avec le glibenclamide augmente progressivement, en fonction du temps. Il convient donc de conclure que le foie libère du glucose. Ce résultat est similaire à celui de Claude Bernard qui, en 1853, a montré que dans les conditions physiologiques, le foie libère du glucose pour répondre à des besoins physiologiques de l'organisme. La production du glucose hépatique et sa libération dans la circulation sanguine seraient dues à l'hydrolyse du glycogène en glucose par l'enzyme glycogène phosphorylase (Madsen, 1991). Comparativement aux rats normoglycémiques témoins, le traitement de rats normoglycémiques avec EAPh aux doses de 800, 1000 et $1200 \mathrm{mg} / \mathrm{kg}$ PC entraîne une diminution dose-dépendante du taux de glucose libéré par le foie. Les résultats obtenus sont conformes à ceux de Takin et al. (2012) qui ont montré que l'extrait aqueux total de Khaya senegalensis (Meliaceae) réduit la libération du glucose hépatique. La libération du glucose par le foie des rats traités avec EAPh à la dose de $1200 \mathrm{mg} / \mathrm{kg}$ PC est sensiblement identique à celle des rats traités avec le glibenclamide à la dose de $10 \mathrm{mg} / \mathrm{kg}$ PC. La réduction de la libération du glucose hépatique en présence de EAPh pourrait s'expliquer par une inhibition de l'enzyme glycogène phosphorylase. 
L'injection de l'alloxane monohydrate aux rats entraîne une augmentation de la glycémie qui passe de 1,02 $\pm 0,05 \mathrm{~g} / \mathrm{L}$ à 2,01 $\pm 0,06 \mathrm{~g} / \mathrm{L}$ et se maintient, traduisant l'installation du diabète expérimental suite à une nécrose des cellules $\beta$ du pancréas. Cette hyperglycémie persiste durant les 28 jours d'expérimentation chez les rats diabétiques non traités. Par contre, lorsque les rats rendus diabétiques sont traités avec l'EAPh ou avec le glibenclamide, l’hyperglycémie diminue significativement et la glycémie tend à revenir à la normale. Le même effet a été observé par Singh et Kakkar (2009) et Ladouari (2012). En effet ces auteurs ont montré que Berberis aristata (Berbridaceae) et Zygophylum album (Zygophyllaceae) réduisent respectivement de $50 \%$ et $33 \%$ l'hyperglycémie chez les rats diabétiques traités pendant 28 jours. Nous pouvons déduire que EAPh, tout comme le glibenclamide, possède des propriétés antidiabétiques. En effet, le glibenclamide, administré à jeun, stimule l'insulinosécrétion, diminue la sécrétion du glucagon, inhibe la libération hépatique du glucose et potentialise les effets de l'insuline au niveau du foie (Jackson et Bressler, 1981).

Chez les rats rendus diabétiques par l'alloxane monohydrate, le taux de glucose hépatique stocké est réduit de $50 \%$, comparativement à celui des rats témoins non diabétiques. La diminution du stockage du glucose hépatique observée chez les rats diabétiques s'expliquerait par une altération de la sécrétion de l'insuline après l'administration de l'alloxane monohydrate. En effet, Elsner et al. (2000) et Shetti et al. (2012) ont montré que l'alloxane altère la sécrétion de l'insuline, ce qui engendre une inhibition de l'activité de la glucokinase. Lorsque les rats diabétiques sont traités avec l'EAPh, après 90 jours, le taux de glucose hépatique stocké augmente significativement et devient sensiblement identique à celui des rats témoins normaux. Ces résultats montrent que l'EAPh favorise le stockage du glucose au niveau du foie. Des résultats similaires ont été rapportés par Kebièche (2009) et Ladouari (2012). Ces auteurs ont respectivement montré que les extraits flavonoïdiques de Ranunculus repens (Ranunculaceae) et l'extrait aqueux de Zygophyllum album (Zygophyllaceae), favorisent le stockage du glucose dans le foie. L'effet de EAPh sur le stockage du glucose hépatique est semblable à celui du glibenclamide. Il peut donc être suggéré que, tout comme le glibenclamide, le mécanisme d'action de EAPh passe par son action directe sur les récepteurs Glut 2 (transporteur du glucose dans la cellule) ou une stimulation des cellules $\beta$ résiduelles du pancréas pour permettre le stockage du glucose.

\section{Conclusion}

La régulation de la glycémie met en jeu le système hormonal ainsi que plusieurs organes (pancréas, foie, rein). Cette régulation fait partie des 
processus de maintien de l'homéostasie au sein de l'organisme. L'extrait aqueux de Pseudarthria hookeri (EAPh) inhibe la libération du glucose hépatique (glycogénolyse) chez les rats normoglycémiques et réduit significativement l'hyperglycémie chez les rats rendus diabétiques. Par ailleurs, EAPh favorise le stockage du glucose dans le foie (glycogenogenèse) des rats rendus diabétiques par l'alloxane monohydrate. Ces résultats prouvent bien l'efficacité de Pseudarthria hookeri (EAPh) dans le traitement du diabète.

\section{References:}

Ambe G-A., Malaisse F., 2000. Les plantes utilisées dans la médecine et la pharmacopée traditionnelles d'une population Malinké en Côte d'Ivoire. Rev Méd Pharm Afr, 14 : 121-130.

Beer A., 1852. Bestimmung der Absorption des rothen Licht in farbigen Flüssigkeiten. Annal en der Physik und Chemie, 86 : 78-88.

Claude B., 1853. Recherche sur une nouvelle fonction du foie, considéré comme organe producteur de matière sucrée chez l'homme et les animaux. Thèse de doctorat de Zoologie de la Faculté des Sciences de Paris (France), $99 \mathrm{p}$.

Elsner M., Tiedge M., Guldbakke B., Munday R., Lenzen S., 2002. Importance of the GLUT 2 glucose transporter for pancreatic beta cell toxicity of alloxane. Diabetologia, 45 : 1542-1549.

Jackson J. E. et Bressler R., 1981. Clinical pharmacology of sulphonylurea hypoglycemic agents. Part I. DRUG ; 212 : 211-245.

Kebieche M., 2009. Activité biochimique des extraits flavonoïdiques de la plante Ranunculus repens L. : effet sur le diabète expérimental et l'hépatotoxicité induite par l'Epirubicine. Thèse de doctorat de Biochimie de l'Université Mentouri Constantine (Algérie), 124 p.

Ladouari A., 2013. Effet du décocté de Zygophyllum album Coss (Zygophylaceae) sur le diabète et sur le stress oxydant associé. Thèse de doctorat de Pharmacie de l'Université d'Alger (Algérie), 81 p.

Madsen N.B., 1991. Glycogen phosphorylase and glycogen synthetase; in kuby. A study of Enzymes, 2 : 139-158.

Moussard C., 2006. Biochimie structurale et métabolique, De Boeck, 187 p.

Shetti A. A., Sanakal R. D., Kaliwal B. B., 2012. Antidiabetic effect of ethanolic leaf extract of Phyllantus amarus in alloxan induced diabetic mice. Asian Journal of Plant Science and Research, 2(1) : 11-15.

Singh J., Kakkar P., 2009. Antihyperglycemic and antioxidant effect of Berberis aristata root extract and its role in regulating carbohydrate metabolism in diabetic rats. Jour Ethnopharmacol. 123 (1) : 22-26.

Takin M., Ahokpe M., Zohoun L., Assou E., Aivodji N., Agossou E., Sezan A., 2014. Effect of total Khaya senegalensis (Meliaceae) barks extracts on 
hepatic liberation of glucose. National Journal of Physiology, Pharmacy and Pharmacology, 4 (2) : 112-117.

Trinder P., 1969. Determination of glucose in blood using glucose oxidase with an alternative oxygen acceptor. Ann Clin Biochem, 6 : 24-27.

Whiting D. R., Guariguata L., Weil C. S. J., 2011. IDF Diabetes Atlas : Global estimates of the prevalence of diabetes for 2011 and 2030. Diabetes Research and Clinical Practice, 94 : 311-321. 